\title{
Asignaciones familiares, licencias e infraestructura de cuidado.
}

\author{
Avances en la intervención judicial \\ Family subsidies, maternity leave and the care infrastructure: improvements in the judges' view.
}

\author{
Pilar Arcidiácono (UBA-CONICET) \\ pilar.arcidiacono@gmail.com) \\ Ana María Bestard \\ (UBA-CONICET) \\ anamariabestard@yahoo.com.ar) \\ Rocío Riesco (UBA) \\ rocio_riesco@hotmail.com \\ Carla Zibecchi (UBA-CONICET) \\ carlazibecchi@hotmail.com
}

Fecha de recepción:

30.4.2015

Fecha de aprobación:

24.7.15

\begin{abstract}
Resumen
Parte del debate feminista moderno ha girado en torno a las licencias por maternidad, el sistema de asignaciones, los servicios y la infraestructura de cuidado. En América Latina diversos actores (el poder legislativo, las organizaciones de derechos humanos y de mujeres y más incipientemente el propio poder judicial) se proponen modificar la extensión, ampliar las coberturas, extenderlas a la paternidad y a los casos de adopción de niños y niñas. En este escenario, este artículo recorre parte de estos debates en el poder judicial que, en general, constituye un actor menos explorado por las ciencias sociales. Para eso se revisan dos casos judiciales en la Argentina que resultan representativos de algunas de las discusiones vinculadas con el cuidado y la naturalización de la división sexual del trabajo -en particular, a través de las vías clásicas de demandas relacionadas con las licencias por maternidad y las guarderías o salas maternales-.
\end{abstract}

Palabras clave: cuidado, poder judicial, empleo, asignaciones familiares, licencias familiares.

\footnotetext{
Abstract

Part of the modern feminist debate has revolved around maternity leave, the system of subsidies and the care services and infrastructure. In Latin America, different actors
} 
(the legislative branch, human rights organizations, women organizations and more incipiently the judicial branch itself) propose to modify the extension and coverage, extending it to paternity and to the cases of children adoption. In this scenario the article covers these debates in the judicial body that in general is an actor less explored by the social sciences. Because of that, the article reviews two judicial cases in Argentina that illustrates some of the discussions related to care particularly through the classic ways of lawsuits related to maternity leave and childcare services.

Keywords: care, judicial branch, employment, subsidies, parent leaves.

\section{Introducción}

Los sistemas de licencias, asignaciones familiares e infraestructura de cuidado juegan un papel central en la disponibilidad de dinero, tiempo y recursos de las familias para el cuidado de niños y niñas pequeños. De modo que actualmente el Estado cumple funciones fundamentales en calidad de empleador en el sector público y de regulador de las relaciones laborales tanto en el sector público como privado, principalmente a través de la Ley de Contrato de Trabajo (LCT) y estatutos específicos (como la reciente normativa de las trabajadoras de casas particulares).

Más particularmente, en la Argentina se verifican los siguientes fenómenos en lo que refiere a la regulación del cuidado: por un lado, en términos normativos se centra principalmente la protección en torno al cuidado de la madre trabajadora (asalariadas registradas) dejando excluidos a grandes sectores de la población, tales como trabajadoras no registradas en el sector formal e informal. En segundo término, la oferta de servicios de cuidado para la primera infancia presenta déficits importantes, al mismo tiempo que se encuentra estratificada en términos socioeconómicos, corporativos -según el estatus laboral de los progenitores- y territoriales -de acuerdo al lugar de residencia-.

En función de esta problemática, este artículo se propone destacar parte del debate feminista moderno en torno a las licencias por maternidad, el sistema de asignaciones y los servicios y la infraestructura de cuidado. Cabe señalar que la discusión en América Latina se ha actualizado a partir del impulso de diversos actores (nuevos proyectos legislativos, organizaciones de derechos humanos, de mujeres y más incipientemente el 
propio poder judicial) que se proponen modificar la extensión, ampliar las coberturas, extenderlas a la paternidad y a los casos de adopción de niños y niñas. Estos debates serán problematizados a través de la revisión de dos casos judiciales en la Argentina sobre algunas de las cuestiones vinculadas con el cuidado -en particular, a través de las vías clásicas de demandas relacionadas con las licencias por maternidad y las guarderías o salas maternales-.

\section{Desarrollo}

\section{Actores que impulsan el debate: la regulación del cuidado}

El cuidado es un término complejo y polisémico que involucra dimensiones materiales, culturales, simbólicas y subjetivas. Sin embargo, existen ciertos consensos conceptuales, más allá de la diversidad de matices, que indican la importancia de comprender por cuidado a un conjunto de actividades necesarias para satisfacer las necesidades básicas para la existencia y desarrollo de las personas. Se puede decir, entonces, que se trata de actividades que sustentan a las personas, en el sentido de otorgarles los elementos fisicos y simbólicos que les permiten vivir en sociedad. Además, existen diversos tipos de cuidado, considerando que existe tanto el cuidado directo (la actividad interpersonal de cuidado) y la provisión de las precondiciones para que ese cuidado pueda realizarse (el trabajo doméstico necesario para proveer el ámbito y los instrumentos de cuidado).A su vez, las necesidades de cuidado pueden satisfacerse en el ámbito familiar, en el mercado o a través del Estado (Pautassi, et al.: 2013.a).

Desde principios de los años sesenta del siglo veinte, feministas militantes de distinto cuño y teóricas del feminismo advertían diversas problemáticas asociadas con lo que hoy se denomina cuidado: la división sexual del trabajo, el trabajo doméstico no remunerado, el trabajo reproductivo, el trabajo de las amas de casa, el salario de la ama de casa, entre otras acepciones. Actualmente, diversas disciplinas de las Ciencias Sociales, tributarias de esos enfoques feministas y estudios de género, lo han construido como un tema de investigación.

Frecuentemente se destaca que la distribución social injusta del cuidado podría explicar las dificultades que enfrentan las mujeres para avanzar en los más variados espacios y las razones por las que se encuentran segregadas. Se observa que el cuidado es un condicionante y determinante de la inserción laboral que pueden desarrollar las mujeres; en el desarrollo de sus carreras profesionales; en el uso del tiempo y del espacio urbano que ellas realizan, en las oportunidades de participación política y social, en los círculos viciosos de pobreza en el cual se sumergen los hogares pobres y en la intermitencia que caracteriza las trayectorias laborales de las mujeres menos educadas; en la modalidad bajo la cual se accede a la cobertura previsional cuando llegan a la vejez. ${ }^{2}$ 
Entre las múltiples ramificaciones de la problemática del cuidado, se señala que disidencias conceptuales sobre el rol social de la maternidad, el embarazo, la lactancia y el cuidado de niños y otros familiares, y la articulación de todos estos momentos de la vida de las mujeres con el trabajo remunerado explican los diversos abordajes propuestos desde el feminismo a la hora de pensar aspectos como las licencias parentales, las asignaciones y las políticas e infraestructura para el cuidado de los niños. ${ }^{3}$ En este sentido, la mayoría de las legislaciones latinoamericanas consagran licencias maternales de distinta extensión para trabajadoras formales y licencias para progenitores varones muy breves (Bergallo y Gherardi 2008).

En la actualidad, existen debates sobre cuidado que fueron permeados por acciones de incidencia de diversas organizaciones de derechos humanos, la presentación de proyectos legislativos, el debate académico y, en menor medida, las manifestaciones volcadas por los jueces en sus resoluciones. A continuación, se presentan dos casos a través de los que se pueden observar las respuestas que los tribunales han brindado a temáticas de licencias, infraestructuras y asignaciones. Sin ser casos considerados litigios estratégicos, los fallos analizados dan cuenta de diversos abordajes sobre la problemática "en el fondo y en la forma”, representando diferentes jurisdicciones e instancias judiciales. A partir de los mismos este artículo aborda: las particularidades de cada uno; el lugar asignado al cuidado y a los diversos formatos de maternidad; el modo en que los magistrados fundaron sus decisiones y el alcance de las mismas; entre otros aspectos relevantes.

Para efectuar esta interpretación de los fallos, nos guiamos por cuatro supuestos fundamentales: (I) reconocer que el derecho, como dispositivo, ha sido un mecanismo de conservación de las condiciones y de las consecuencias de desigualdad, y que las construcciones jurídicas han organizado identidades sexuales y de género, prescribiendo responsabilidades, roles, entre otros elementos; (II) si bien el derecho y las instituciones jurídicas se han definido como neutrales en términos de género, en realidad, son una construcción cultural y política que fortalece y naturaliza la masculinidad y la heterosexualidad (Motta y Sáez 2008); (III) existe cierta recursividad entre los cambios en las relaciones de cuidado y el sistema legal: transformaciones en las relaciones de cuidado pueden tener potencialidad para promover cambios en las intervenciones judiciales y viceversa (Zelizer 2009); (IV) se considera a los jueces como intérpretes y actores que despliegan una actitud recreativa y creadora de sentido a la norma legal, como parte de un proceso que se adelanta a los problemas, ergo, el contenido de los fallos es resultado de un proceso incesante de atribución de sentido a las normas y principios, selección de reglas o principios aplicables de acuerdo a esa atribución de sentido y otra multiplicidad de operaciones intelectuales en donde convergen factores ideológicos, valorativos y extra normativos (Courtis y Bovino 2001). 


\begin{abstract}
Asignaciones y licencias
La interpretación judicial en la causa "M. V., M. C.Y OTRO S/AMPARO". San Carlos de Bariloche, 11-05-2006. Cámara del Trabajo de Río Negro Sala/Juzgado: III circunscripción

El siguiente fallo recupera el caso de la señora M. C. M.Villarroel y el señor J.A. R. que promueven una acción de amparo con la finalidad de que se le otorgue la licencia por maternidad en el establecimiento donde trabaja, "Hotelera Lago Sur S.R.L.", a raíz de haber recibido un niño recién nacido en guarda para adopción. Habiendo solicitado dicha licencia a la empleadora, ésta informó sobre la imposibilidad de su otorgamiento en razón de que no existe norma vigente que contemple la misma para el caso de adopción.
\end{abstract}

Recordemos que la Asignación por Maternidad, más conocida como "licencia por maternidad", consiste en el pago de un monto igual a la remuneración bruta que le hubiera correspondido percibir a la trabajadora durante el período de licencia legal en el empleo con motivo nacimiento de su hijo/a. La licencia por maternidad es percibida por las trabajadoras en relación de dependencia o beneficiarias de una Aseguradora de Riesgos del Trabajo y cuando la trabajadora se desempeña en más de un empleo. En cada caso, tiene derecho a la percepción de esta Asignación. ${ }^{4}$ Para acceder a la misma la trabajadora debe contar con una antigüedad mínima y continuada de tres meses en cada uno de los empleos a la fecha de inicio de la licencia. ${ }^{5}$ No obstante, hasta el momento, la normativa vigente en la Argentina no extiende la licencia a casos de adopción.

En el trámite judicial del amparo aquí analizado se requirió un informe a Hotelera Lago Sur S.R.L y a ANSES. Se recibió únicamente respuesta de este último organismo que remitió a las disposiciones legales vigentes que no contemplan la Asignación por Maternidad en el supuesto de adopción. El propio organismo sostiene que únicamente se encuentra contemplada la Asignación Familiar por Adopción, prestación que se abona una vez que se presenta la sentencia definitiva que la reconoce y por única vez (al igual que en caso de nacimiento) pero que habilita luego a la Asignación por Hijo (mensual). En la instancia de apelación, la Cámara finalmente resolvió hacer lugar a la acción de amparo promovida. Declaró que la señora M. C. M.Villarroel tiene derecho a gozar de licencia de maternidad con motivo del otorgamiento en guarda para adopción del niño. Asimismo, señaló que le corresponde el derecho a percibir las asignaciones que confieren los sistemas de seguridad social.

El fallo se vale de diversos instrumentos de derechos humanos y de argumentos próximos a la perspectiva de género y a la protección de los derechos de los niños, niñas y adolescentes. Dichos argumentos, en apretada síntesis, sostienen que la necesidad de cuidado de la madre y del recién nacido trasciende las diferencias entre maternidad biológica y adoptiva. Es decir, se sostiene que no hay justificación para defender la omisión 
por parte del Estado en materia de licencias por maternidad en caso de adopción y que constituye un deber insoslayable de los jueces tutelar dichos derechos esenciales. Incluso, en el fallo se afirma que

la equiparación de la maternidad biológica y maternidad adoptiva no admite punto de discusión alguno desde la lógica de la razón y el sentido común y la ausencia de una norma expresa en tal sentido dentro de nuestra legislación no impide que se aplique en ambos casos el mismo régimen laboral, correspondiendo al órgano judicial cubrir el vacío legal en la materia" (Considerando $5^{\circ}$ del Fallo).

La argumentación del tribunal parte de derechos fundamentales consagrados en la Constitución Nacional (CN) y en algunos Tratados Internacionales de Derechos Humanos (TIDH) con jerarquía constitucional ${ }^{6}$ como en otros instrumentos que no gozan de la última. Por un lado, se alude a la garantía de igualdad ante la ley del artículo 16 $\mathrm{CN},{ }^{7}$ pues en el fallo se cataloga como "absolutamente injusta $y$ discriminatoria la distinción entre ambas (madre biológica y madre adoptiva) que hace la autoridad de aplicación”. Por otro lado, se menciona el derecho a la protección del trabajo en sus diversas formas del artículo 14 bis $\mathrm{CN}^{8}$ Asimismo, se hace referencia al amparo de la familia y el cuidado de los hijos establecidos expresamente como derechos de los ciudadanos en los artículos 31 a 33 de la Constitución Provincial de Río Negro, y se aclara en ese sentido:

(...) tomado esto no sólo como obligación de los padres sino con el carácter de responsabilidad social, debiendo entenderse que el ámbito de dicho ordenamiento no se limita a la familia biológica sino que se extiende a los vínculos adoptivos" (Considerando $3^{\circ}$ del Fallo).

El argumento que atraviesa la decisión, desde el comienzo hasta el final, es el de la igualdad real de oportunidades y de trato, aunque en el fallo no hay una enunciación expresa de esta igualdad material ni un desarrollo robusto de la misma. El fallo únicamente cita el art. $16 \mathrm{CN}$ y luego los TIDH con jerarquía constitucional que aluden al principio de la no discriminación entre la madre biológica y la adoptiva.

Si bien el fallo sólo menciona el art. 16 CN en materia de igualdad a nivel constitucional, en verdad, aplica el concepto de igualdad material, cuestión realmente importante. Pues, el tribunal hace operativa la norma constitucional y el derecho internacional de los derechos humanos (Bidart Campos 2004). Asimismo, cita la IV Conferencia Mundial sobre la Mujer (Beijing 1995) que reconoce la importancia social de la maternidad y la función de los padres en la familia que requiere la responsabilidad compartida entre 
hombres y mujeres y la sociedad en su conjunto. Agrega el Convenio $N^{\circ} 156$ sobre la "Igualdad de oportunidades de trato entre trabajadores y trabajadoras con responsabilidades familiares" (OIT) (arts. 3, 7, 8). Por su parte, la fundamentación concluye en que el objetivo común de las normas internacionales citadas apunta a la implementación de medidas que le permitan a los trabajadores satisfacer adecuadamente las tareas y las obligaciones familiares, protegiendo especialmente a las mujeres de cualquier situación discriminatoria por su condición de madres.

Cabe señalar que la Recomendación 191 de la OIT (2000), que no se contempla en el fallo, procura extender la duración de la licencia por maternidad a 18 semanas, prevé una prolongación en caso de nacimientos múltiples y deja que la mujer elija libremente cuándo tomará la parte no obligatoria de su licencia de maternidad, antes o después del parto. Esta recomendación incluye también aspectos vinculados con las prestaciones, su financiación, la protección de la salud, las madres lactantes y otras licencias relacionadas (licencia parental y licencia de adopción, entre otras).

Concordantemente, en algunos países de la región ya se reconoce la licencia por adopción (Pautassi y Rico: 2011: 3). En casi todos los casos, la protección está dirigida a las madres trabajadoras formales y en relación de dependencia, y las disposiciones establecen el fuero maternal que las protege contra los despidos sin causa justificada durante el embarazo, puerperio y lactancia.

El caso argentino presenta un retraso importante en este punto, razón que explica el surgimiento del presente caso judicial. En lo que refiere a las licencias por adopción, sólo algunos convenios colectivos de trabajo contemplan este derecho, como, por ejemplo, la Administración Pública y aquellas normas que regulan al personal docente. Es importante aclarar que la Ley de Contrato de Trabajo no establece ninguna licencia por adopción; tampoco lo hace la nueva normativa sobre trabajadoras de casas particulares ni la Ley de Trabajo Agrario ${ }^{9}$ (Aulicino et al.: 2013: 26). ${ }^{10}$ En la actualidad existen algunos proyectos en el Poder Legislativo para avanzar no sólo en materia de madres y padres adoptantes sino en la ampliación de licencias para madres y padres en casos de nacimiento.

En el mismo fallo puede identificarse un tratamiento diferente cuando implica el otorgamiento de la licencia y de la asignación familiar. En el primer caso se trata de un vacío normativo (no se contemplan licencias por maternidad en casos de adopción ya sea en el momento de la guarda o de la sentencia definitiva). En este marco, el tribunal reacciona aplicando fundamentalmente un conjunto de principios y normas de carácter interno e internacional para resolver la discriminación, razón por la cual el presente fallo es mencionado en algunas otras sentencias y proyectos legislativos que proponen la extensión de la licencia en estos casos. ${ }^{11}$ 
Ahora bien, en el segundo caso -Asignación Familiar por Adopción- no existe un vacío normativo sino que la norma vigente establece la instancia de la sentencia como aquella que habilita a la percepción de la prestación, no siendo la guarda suficiente para otorgarla. En este fallo se aplica la prestación sin ley que así lo disponga desde la guarda con fines de adopción, apelando a la no distinción entre maternidad biológica y adoptiva En el caso de la primera, la asignación se cobra inmediatamente posterior al nacimiento (Asignación por Nacimiento) lo que luego habilita al cobro mensual de la Asignación por Hijo.

Más allá del poder judicial: el problema de las licencias por maternidad, las asignaciones familiares y el cuidado

Desde la década de 1970 las concepciones de las feministas en torno a las licencias por maternidad y los permisos parentales, en general, se han dividido en diversas posiciones. En una primera época el debate se dio en el contexto de la tensión entre el modelo de trato igualitario y las defensoras del trato específico para capturar las diferencias entre los sexos (Bergallo y Gherardi 2008). ${ }^{12}$ Actualmente, la discusión más moderna se ha sofisticado al punto de ubicar el tema de las licencias parentales en uno de los problemas vinculados a cuestiones más amplias que implica una revisión a las nuevas formas de familias - por ejemplo, el matrimonio igualitario- y temas clásicos - pero aún no resueltos- como es el caso de la adopción.

Ahora bien, ¿Cómo se instala este debate en Argentina? Al igual que en otros países de América Latina -más allá de la diversidad de enfoques y matices- el debate de las licencias y la infraestructura de cuidado -tema que abordaremos en la siguiente sección- se enmarca y se relaciona con una diversidad de temas: la división sexual del trabajo doméstico, la regulación del cuidado, la mayor productividad de los trabajadores y trabajadoras, la promoción de la inserción laboral de las mujeres, las políticas de conciliación familia y trabajo, la responsabilidad empresarial, la corresponsabilidad entre progenitores de ambos sexos, entre otros aspectos.

En este sentido, puede decirse que dos movimientos ocurridos durante los últimos años colaboraron para que este debate se haga presente. En primer lugar, se observa el aporte de expertas en estudios de género e investigadoras feministas que fueron desarrollando nuevas y renovadas líneas de estudios con la llegada a la democracia en la Argentina en universidades y diversos centros de estudios.

En segundo término, el debate se actualizó a partir de la presentación de proyectos legislativos que proponen extender licencias por maternidad y paternidad. ${ }^{13}$ Es decir, la cuestión del cuidado y la conciliación ha ido permeando la agenda legislativa, en la que se pueden identificar numerosas iniciativas traducidas en proyectos de ley que buscan ampliar las licencias existentes o crear nuevas, ya sean por maternidad o parentales, 
así como impulsar diferentes acciones vinculadas al reconocimiento de las tareas de cuidado y a la ampliación de la oferta de servicios de cuidado (Rodríguez Enríquez y Pautassi 2014).

Ahora bien, a fin de comprender la resolución del tribunal de justicia recién examinada, a continuación se desarrolla sucintamente la dinámica del sistema de asignaciones familiares en Argentina.

El sistema de asignaciones familiares se implementa en la Argentina desde 1957 y hacia 1973 se consolidó el proceso de inclusión de todos los sectores de trabajadores formales al sistema, con el objetivo de contribuir al sostenimiento y expansión del núcleo familiar de los trabajadores formales a través de transferencias de ingresos que se realizaban por intermedio del sistema de seguridad social. ${ }^{14}$

La Asignación por Adopción consiste en el pago de una suma de dinero que abona ANSES en forma directa al trabajador en relación de dependencia, titular de la prestación por desempleo, titular de la prestación de una Aseguradora de Riesgos del Trabajo o titular de la Pensión Honorífica de Veteranos de Guerra del Atlántico Sur, por la adopción de un menor de edad. La misma es abonada sólo a uno de los padres/adoptantes y la solicitud puede realizarse recién una vez transcurridos los dos meses de dictada la sentencia de adopción y hasta dentro de los dos años. Tal como sucede para el resto de las asignaciones (excepto la Asignación por Maternidad o licencia), uno de los requisitos se asocia con el cumplimiento de los topes, tanto individuales o del grupo familiar, establecidos en la normativa vigente a la fecha de ocurrido el nacimiento o de dictada la sentencia de adopción. ${ }^{15}$

Sin dudas, una dimensión de la problemática reside en que no todas las mujeres trabajadoras gozan en igualdad de condiciones de estos beneficios. Las personas que trabajan de manera no registrada o informal, así como quienes están comprendidas bajo las modalidades de monotributo o autónomo (cuentapropistas, profesionales y trabajadoras/ es independientes) quedan por fuera de cualquier régimen de licencias para el cuidado. En la LCT la regulación en torno al cuidado se circunscribe a la protección a la madre trabajadora en el régimen laboral, fundamentalmente en el período de gestación, alumbramiento y lactancia.Además, se observa un conjunto de disposiciones que se refieren casi exclusivamente a los derechos de las mujeres y casi nunca a los de los varones. De esta forma, y para el caso de los trabajadores varones, estas disposiciones parten del supuesto de que existe una mujer que cubrirá las necesidades de cuidado de los hijos. En la misma dirección, este supuesto se encuentra implícito en todos los beneficios que se otorgan: licencias por enfermedad, acceso a guarderías, período de excedencia (Pautassi et al.: 2004). 


\section{Servicios de salas maternales}

La interpretación judicial: "DELLEDONNE MARCELA ALEJANDRA C/ MINISTERIO DE SEGURIDAD - POLICIA DE LA PROVINCIA S/AMPARO" Mar del Plata, 13-05-2004. Juzgado Contencioso Administrativo de Mar del Plata.

El siguiente fallo se origina a partir de una acción de amparo que interpone Marcela Delledonne contra la Policía de la Provincia de Buenos Aires por la arbitraria denegación del reintegro por guardería. Solicita que se ordene al ente demandado otorgar dicho beneficio previsto por la Ley 11.501 (incorporado al Decreto ley 9550/80, de la ley Orgánica de la Policía de la Provincia de Buenos Aires en su art. 116 inc. d) apartado $\left.7^{\circ}\right)$, y reintegrar lo que a la fecha se le adeudaba por ese concepto. ${ }^{16}$

Marcela Delledonne se desempeñaba como policía de la Provincia de Buenos Aires con la jerarquía de Suboficial Inspector. Originaria de la ciudad de La Plata, donde residía con toda su familia, es trasladada a Mar del Plata para desempeñarse en una Comisaría del municipio de General Pueyrredón. Desde el nacimiento de sus dos hijos y debido a sus horarios de trabajo, ella debía dejarlos en un jardín maternal. Sin embargo, no exise en la ciudad de Mar del Plata una guardería estatal gratuita. Por otro lado, en el momento de la presentación del caso se encontraba embarazada de su tercer hijo, por lo que necesitaba mandar a los tres niños a la guardería para poder trabajar. En el presente fallo, el motivo invocado por el Poder Ejecutivo de la Provincia de Buenos Aires para desechar el reclamo es la falta de reglamentación del beneficio por guardería.

El fallo resolvió hacer lugar a la acción de amparo promovida declarando manifiestamente ilegítima la demora del Ejecutivo en la reglamentación del artículo mencionado y, en consecuencia, lo condenó a hacerlo dentro del plazo de treinta días corridos. Asimismo, le ordenó que, en el plazo de treinta días a computar desde que se dicte la reglamentación indicada, resuelva el reclamo particular de la demandada, bajo apercibimiento de astreintes. ${ }^{17}$

El fundamento central del fallo es la omisión inconstitucional del Ejecutivo en reglamentar la ley que consagraba el derecho a la guardería en cuestión. Tanto la Fiscalía de Estado como la Dirección de Fiscalización de Asignaciones de la Policía se habían expedido en contra de lo solicitado por Delledonne (Considerando $4^{\circ}$ del fallo). Se revierte la negativa de la Administración en base a que

luego de 10 (diez) años de vigencia de la ley el Poder Ejecutivo provincial no puede seguir demorando su aplicación con la excusa de la falta de reglamentación que a él mismo le corresponde hacer del beneficio" y que frente al reclamo "el Poder Judicial no puede permanecer ajeno 
por encontrarse en juego elementales principios constitucionales (Considerando $5^{\circ}$ del fallo).

Para el juez, la omisión reglamentaria importa la violación no sólo de la Ley provincial 11.501 sino también del art. 144 inc. 2 de la Constitución de la Provincia de Buenos Aires, que le atribuye al Poder Ejecutivo la competencia de facilitar la ejecución de las leyes.

Cabe destacar que si bien el fallo se vale de la inconstitucionalidad por omisión, este instituto no fue receptado por la Constitución de la Provincia de Buenos Aires ni por la Constitución Nacional, aunque la Suprema Corte de Justicia de la Provincia de Buenos Aires -como señala el mismo fallo comentado- se ha pronunciado en diversas oportunidades poniéndole límites a la inactividad de la administración provincial, con fundamento en que las omisiones configuradas en los casos analizados implicaban supuestos de inconstitucionalidad. ${ }^{18}$ Hemos destacado, en el análisis del fallo anterior y reiteramos en el análisis del fallo actual, la notable influencia de la reforma constitucional de 1994 respecto de la exigibilidad de los derechos sociales y del contenido material de la garantía de igualdad y no discriminación (art. 75 incs. 22 y $23 \mathrm{CN}$ ), que debe regir el goce de los derechos sociales. Sin embargo, a diferencia del caso anterior -donde la sentencia sostuvo su argumentación más allá del andamiaje de los derechos humanos, apoyándose en cuestiones que hacen a los problemas de cuidado-; en este fallo, el tipo de estrategia utilizada para sostener la argumentación está centralmente basada en cuestiones de índole procesal.

Además, como se verá a continuación, refleja uno de los problemas centrales de las cuestiones que hacen a la provisión del cuidado en la primera infancia.Valga aclarar también que, como el caso anterior, ambos abordan la problemática de cuidado de trabajadoras asalariadas y registradas que se suponen que tienen mayor acceso -sea a través del sistema de protección social que le brinda su estatus laboral de trabajadoras asalariadas registradas, o en otros casos a través de la capacidad adquisitiva de su salario a servicios e infraestructura de cuidado- en comparación con las trabajadoras no registradas, del sector informal, las monotributistas y las que se dedican exclusivamente al trabajo de cuidado de sus familias (es decir, las denominadas “amas de casa”).

El otro lado de la justicia: déficits de servicios e infraestructura de cuidado El análisis precedente en relación al servicio de guardería o salas maternales debe ser comprendido en el marco de una problemática más amplia vinculada con la organización social del cuidado. En forma sucinta se puede decir que los principales servicios, instituciones y arreglos institucionales que constituyen la oferta de cuidado y que intervienen en la distribución social del cuidado en Argentina son: (I) las que se vinculan con el sistema educativo (en especial, salas maternales y jardines de infantes para la primera 
infancia y educación formal del nivel primario), jardines comunitarios e intervenciones asistenciales por parte de algunos Estados locales; (II) servicios contratados en el mercado, entre los cuales se destaca la contratación de trabajadoras de casas particulares que constituye una de las principales estrategias de cuidado que implementan las familias de ingresos medios y altos para el cuidado de sus hijos y, a su vez, constituye la principal ocupación de las mujeres pobres; (III) el sistema de protección de la madre trabajadora formal asalariada.

Como se mencionó, el caso analizado se vincula precisamente con el sistema de protección de la madre trabajadora formal asalariada, principalmente con la infraestructura y servicios de cuidado que deberían proveer establecimientos laborales y empleadores. En este sentido, no puede pasarse por alto que en Argentina el Estado cumple dos funciones fundamentales; por un lado, en calidad de empleador en el sector público (poderes ejecutivo, legislativo, judicial en las jurisdicciones nacionales, provinciales y municipales; establecimientos educativos de gestión estatal, etc.). Por otra parte, en calidad de regulador de las relaciones laborales tanto en el sector público como privado, principalmente a través de la Ley de Contrato de Trabajo (LCT) y estatutos específicos. También los sindicatos son actores centrales para impulsar estos derechos y beneficios vinculados con la regulación del cuidado en los procesos de negociación colectiva. Sin embargo, existe una baja presencia de estos temas en las negociaciones colectivas debido a múltiples factores: el no cumplimiento de la Ley de Cupo Sindical, ${ }^{19}$ cuyo objetivo es asegurar las candidaturas de mujeres; la reducida presencia femenina entre los dirigentes sindicales y en las instancias de negociación y de toma de decisión; la escasa capacitación de los trabajadores de ambos sexos para negociar con los empleadores cláusulas relativas a la promoción de la igualdad de oportunidades y de trato; y la aún baja priorización del tema en las estrategias sindicales (Zibecchi: 2014: 37). De todas formas, haciendo hincapié en este caso en particular valga señalar como salvedad que debido a la falta de sindicalización que aún mantiene esta fuerza, no existen espacios formales de canalización para esta y otras cuestiones que hacen a sus condiciones laborales y salariales (CELS 2007: 140).

Por su parte, los empleadores (estatales o privados) son también actores importantes en tanto pueden llevar adelante acciones relevantes para articular las responsabilidades laborales con las familiares, acciones y políticas de conciliación familia trabajo. ${ }^{20}$ Estas acciones buscan atenuar lo que se denomina "conflicto familia y trabajo" que atraviesan particularmente las trabajadoras-madres, debido a que son actualmente quienes se ocupan principalmente de las responsabilidades de cuidado. Por un lado, como empleadores deben cumplir con la normativa vigente que establece la LCT y los convenios colectivos de trabajo. La LCT establece el marco normativo de protección de derechos para los trabajadores registrados y en relación de dependencia en todo el territorio del país. Este piso mínimo también puede ser ampliado por los convenios colectivos de trabajo 
de cada sector. Por otra parte, pueden asumir otras acciones que tienen la capacidad de otorgar beneficios adicionales o la ampliación de los beneficios que establece la normativa vigente. ${ }^{21}$ No obstante, y como vimos en el análisis precedente del fallo, el avance en estas líneas de intervención aún está en un estado embrionario en términos normativos. La LCT impone a los empleadores la habilitación de salas maternales y guarderías en función del número de trabajadoras ocupadas (LCT, art. 179); no obstante, la norma nunca fue reglamentada y tiene poca operatividad. La Ley 11.317 estableció un cupo de 50 trabajadoras mayores de 18 años por establecimiento para que rija la obligación de instalar salas maternales y guarderías. ${ }^{22}$ Posteriormente, también la Ley 20.582 dispuso la instalación de jardines maternales zonales. ${ }^{23}$ Sin embargo, estas disposiciones no han sido reglamentadas ni actúan coordinadamente, por lo que no se ha desarrollado ningún sistema que garantice el cuidado de los niños durante la jornada de trabajo. Siguiendo el análisis efectuado por Pautassi et al. (2004), la estrategia de "conciliación" de responsabilidades a través de las guarderías y jardines de las empresas donde trabajan las madres ocupa un lugar residual. En la misma dirección, en las grandes y medianas empresas relevadas por un diagnóstico efectuado por ELA, CIEP y ADC, se destaca que ninguna contaba con salas maternales y que optaban por dar subsidios para que sus empleados puedan acceder a ellas (Rodríguez Enríquez y Pautassi: 2014).

Ahora bien, la problemática de las salas maternales o guarderías debe ser entendida en el marco de un espectro más amplio asociado con el déficit de servicios e infraestructura de cuidado acorde con las necesidades de las familias: hoy existe una fragmentación de la oferta en distintos sectores (el sistema educativo del nivel inicial de gestión estatal, de gestión privada, los programas de atención integral y el ámbito comunitario como proveedor de servicios), ${ }^{24}$ en el nivel inicial de gestión estatal se observa escasa cobertura de establecimientos que ofrezcan servicios de jornada completa (los que existen son muy demandados) y baja tasa de escolarización en las edades no obligatorias; las propias características de la oferta de servicios de cuidado (debilidad de la oferta de gestión estatal y segmentación de la oferta privada) implica que el acceso a los mismos resulta desigual según niveles de ingreso y zonas de residencia, entre otras cuestiones.

\section{Conclusión}

Los temas asociados con el cuidado han tenido un sostenido y renovado impulso desde el ámbito de las organizaciones de derechos humanos -especialmente pero no únicamente, de mujeres- y desde la producción académica. Durante los últimos años, en diversos países de América Latina, estos temas fueron adquiriendo mayor protagonismo en la escena pública captando la atención -con diversidad de posiciones y matices- de legisladores, de algunos sindicatos y también del poder judicial. 
El debate que aquí se recupera presenta nuevas y viejas tensiones que asumen la forma de una suerte de "péndulo" entre las condiciones de trabajo exigida por los empleadores y la demanda de protección de los trabajadores y de las trabajadoras madres; entre la necesidad y deseo de las mujeres por incorporarse en el mercado laboral y las demandas del mundo familiar (conflicto familia y trabajo); entre el desarrollo laboral de las mujeres y el cuidado de los hijos; entre el derecho al trabajo de los progenitores y el derecho al cuidado de los hijos, entre otras.

Como se señaló anteriormente, en la Argentina existen dos grandes ejes relacionados con la normativa referida a la articulación entre la vida laboral y la vida familiar. El primero de ellos se centra en el período de gestación, alumbramiento y lactancia. El segundo abarca un conjunto de disposiciones que se refieren casi exclusivamente a los derechos de las mujeres asumiendo siempre doble función de trabajadoras-madres, que por un lado, naturaliza aún más la idea de que hay una trabajadora madre que cubrirá las necesidades de cuidado de los hijos y por el otro, limita la participación de los progenitores varones. Además, allí se puede visualizar que lo protegido y reglamentado para las mujeres se relaciona con su responsabilidad sobre el mundo privado, más que sobre el continuum productivo-reproductivo como eje de análisis de relaciones que incluyen a varones y mujeres, o sobre la eliminación de discriminaciones en el mundo de lo público (Pautassi et al., 2004).

En este debate no están ausentes las viejas tensiones que ya se observaban a principios del siglo XX sobre el lugar de la madre trabajadora: resurge la preocupación por la lactancia, la maternidad, la salud del recién nacido, el impacto económico de las medidas protectoras y la reducción de la pobreza, entre otros temas que marcaron la cuestión social de aquel entonces (Staab: 2013).

Entendemos que las regulaciones sobre las licencias, así como todos aquellos beneficios (infraestructura y servicios de cuidado) constituyen formas de obligar a otros actores a afrontar los subsidios cruzados o a asumir ciertos costos ocultos que serían, de otra manera, asumidos sólo por las mujeres. Al menos que estos costos sean reconocidos y que la compensación sea incorporada en la regulación y en el diseño de la política pública, los enunciados vinculados con la protección de las trabajadoras corren el riesgo de no ser más que un "sentimentalismo moralizador" (Rittich: 2012,36). Además, no debe pasarse por alto que la intensa naturalización que aún impera sobre la división sexual del trabajo -que tiene características estructurales- impide que casos como los aquí analizados sea demandados por todos los trabajadores y trabajadoras.

Ambos fallos aquí analizados vienen a mostrar omisiones de prestaciones y servicios para las trabajadoras madres asalariadas. Señalan que más allá de los importantes avances -en materia de Asignación Universal por Hijo para trabajadoras del sector informal y 
para las mujeres que se dedican exclusivamente al trabajo de cuidado de sus familias, la nueva normativa para las trabajadoras de casas particulares, entre otros- la oferta de prestaciones y servicios de cuidado continúa atravesada por la condición salarial de la mujer madre asalariada, de allí que uno de los principales déficits es la exclusión de un gran contingente de trabajadores y trabajadoras para los cuales no existe ningún tipo de protección y cobertura.

Consideramos de relevancia analizar las intervenciones judiciales dado que una de las modificaciones más importantes de la reforma de la CN de 1994 fue la de consagrar el aumento y profundización los derechos fundamentales (entre ellos, los sociales) y la de obligar al Poder Legislativo Nacional a reglamentarlos a efectos de reconocerlos y determinar su alcance. A su vez, este verdadero avance jurídico quedó completado con la constitucionalización y ampliación de la garantía del amparo que la reforma incorporó en el art. $43 \mathrm{CN}$, otorgando al juez una poderosa herramienta para hacerlos efectivos frente a los reclamos particulares, herramienta que con un alcance cada vez más creciente ha utilizado tanto el Poder Judicial Nacional como el local. De ahí en adelante, las intervenciones pueden ser de lo más variadas.

El primer fallo analizado limita su alcance estrictamente al plano individual, aunque viene a dar cuenta de un vacío estructural en el sistema de licencias y asignaciones familiares en casos de adopción, ya que Argentina muestra cierto retraso comparado con varios países de la región. En cambio, en el segundo hay una pretensión diferente aunque, por el momento, incumplida. Con argumentos de índole procesal, se toma como punto de partida un caso individual para abordar una omisión reglamentaria en materia de infraestructura de cuidado y sentenciar la necesidad de regulación del beneficio por guardería de la Ley 11.501. El juez declaró ilegítima la demora del Poder Ejecutivo de la Provincia de Buenos Aires en la reglamentación del mismo y, en consecuencia, lo condenó a que, dentro del plazo de 30 días corridos, logre reglamentar dicha prestación. Sin embargo, hasta la fecha no se ha cumplido con la reglamentación, sólo lográndose la solución con alcance para la demandante. Más allá de que en este segundo caso se trata de un mandato concreto, de alguna manera ambos operan como "señales de alarma" para indicarles a los poderes ejecutivo y legislativo que deben definir un curso de acción frente a un problema que no ha sido considerado.

De todas formas, dado que las temáticas abordadas requieren del involucramiento de múltiples actores y agencias, las sentencias lejos de constituir la culminación del proceso operan como un punto de inflexión que en el mejor de los casos puede inaugurar un nuevo proceso de interlocución (Fairstein et al.2010: 136) o no tener un gran impacto político. Esto se observa más claramente en el caso de las licencias donde aún no se ha reglamentado la normativa. Esa función judicial puede ser provechosa para la efectividad de la acción de gobierno, pero el efecto virtuoso de la "cadena" de casos indivi- 
duales depende de la capacidad de reacción del sistema político y de su plasticidad para entender la serie de litigios como "señal de alarma" y adaptar la agenda en consecuencia (Arcidiácono y Gamallo 2014: 45). Sin dudas, la acción de los tribunales se presenta como una estrategia política abierta, compleja, ambivalente y controvertida (Arcidiácono y Zibecchi 2013: 13) enmarcada en una transformación de la esfera pública, donde la temática del cuidado toma cierto protagonismo.

Por otra parte, las temáticas vinculadas con el cuidado resultan un "prisma" para observar intervenciones judiciales en asuntos que tienen un alto contenido moral. Aun constituyéndose en temas clásicos sobre la problemática examinada (licencias, salas maternales) el cuidado es una relación social que por su propia naturaleza tiene características que la diferencian sustancialmente de otras relaciones. Entre ellas se encuentra el alto componente moral e ideológico que hace que el cuidado aparezca como un acto natural y propio del ser mujer ("buen cuidado", "buena madre", "buena esposa"). No es casual, entonces, que en los casos analizados el lugar de los progenitores varones se encuentra difuso en la mirada judicial, siendo el centro de argumentación la trabajadora madre.

Al comienzo del artículo, se partió de algunos supuestos que implicaban reconocer que si bien el derecho y las instituciones jurídicas se han definido como neutrales en términos de género, fortalecen y naturalizan la masculinidad; constituyen mecanismos de conservación de las condiciones y de las consecuencias de desigualdad; organizan identidades sexuales y de género, prescribiendo responsabilidades y roles, entre otras cuestiones.

Los casos analizados ilustran sobre la intervención del poder judicial que, como cualquier actor político, toma posición, incluyendo o no estos temas, abordándolos de forma más o menos explícita o de manera más velada en sus argumentaciones. Al mismo tiempo, pueden basarse en razonamientos de índole más tradicional con justificaciones más procesales (como el caso de guarderías) o indagando en otros andamiajes más renovados (como el de adopción). Como parte del Estado, el poder judicial es un actor partícipe de las disputas en materia de cuidado, la naturalización de la división sexual del trabajo, el lugar y rol asignado a las trabajadoras madres. 
* Este trabajo fue efectuado en el marco del proyecto: "Derecho al cuidado y provisión de bienestar en la Argentina actual" (UBACyT joven). Secretaría de Ciencia y Técnica de la Universidad de Buenos Aires. Sedes: Facultad de Derecho y Facultad de Ciencias Sociales (UBA). Período 2012-2014. Directora: Carla Zibecchi y codirectora: Pilar Arcidiácono.

${ }^{2}$ Existen investigaciones que explican las razones por las cuales debe considerarse que el acto de cuidar es un trabajo-mayoritariamente efectuado por las mujeres-: implica tiempo, desgaste de energía y genera valor (CEPAL 2009).

${ }^{3}$ Para un desarrollo de estos temas se pueden consultar los trabajos compilados por Laura Pautassi y Carla Zibecchi (2013).

${ }^{4}$ Por ejemplo, algunas feministas liberales, alineadas en una recepción feministas de las propuestas liberales rawlsianas han reconceptualizado algunas de las visiones más antiguas sobre la exigencia de la "neutralidad" estatal sobre el tema. De allí que han adscripto a las reivindicaciones similares a las feministas socialistas a partir del reconocimiento de la importancia de la promoción de la igualdad en la distribución de responsabilidades de cuidado. En cambio, las feministas culturales han revalorizado el rol de la mujer embarazada, su protección como trabajadora y la definición de una serie de beneficios específicos para las mujeres, por ejemplo, reivindicando el "salario de ama de casa" (Bergallo y Gherardi 2008).

${ }^{5}$ En el caso de remuneraciones variables se tiene en cuenta para determinar el monto al promedio de las remuneraciones percibidas durante el período de tres meses anteriores al comienzo de la licencia por maternidad. Fuente: www.anses.gob.ar (consultada en septiembre 2014).

${ }^{6} \mathrm{El}$ art. 75 inc $22 \mathrm{CN}$ enuncia una serie de TIDH (Declaración Americana de los Derechos y Deberes del Hombre; Declaración Universal de Derechos Humanos, Convención Americana de Derechos Humanos, Pacto Internacional de Derechos Civiles y Políticos, Pacto Internacional de Derechos Económicos Sociales y Culturales, Convención de los Derechos del Niño, Convención sobre Eliminación de todas las formas de Discriminación contra la Mujer, etc. ) a los que les asigna jerarquía constitucional y agrega que podrán incorporarse otros tratados con igual jerarquía, pero debiendo ser aprobados a tales efectos, por el Congreso, en un procedimiento agravado con mayorías especiales (dos tercios de los miembros totales de ambas cámaras).

${ }^{7} \mathrm{El}$ art. $16 \mathrm{CN}$ establece que todos los habitantes son iguales ante la ley y admisibles en los empleos sin otra condición que la idoneidad.

${ }^{8} \mathrm{El}$ art. 14 bis $\mathrm{CN}$, en su $3^{\circ}$ párrafo consagra la seguridad social, con carácter integral e irrenunciable, a cargo del Estado, y que la ley establecerá la protección integral de la familia, la compensación económica familiar, entre otros derechos.

${ }^{9}$ Ley de Trabajo Agrario N ${ }^{\circ}$ 26.727, sancionada en 2011.

${ }^{10}$ En el caso de la Administración Pública Nacional, el Convenio Colectivo del Sector Público dispone ya a partir de la tenencia con fines de adopción que se le concede licencia con goce de haberes a la mujer por un término de 100 días corridos (art. 121).En el caso de los docentes, el Estatuto prevé para las madres una licencia por adopción (de niños/as hasta 7 años de edad) de 90 días. También el Convenio Colectivo que rige para ANSES establece 45 días corridos de licencia por adopción, con posibilidad de ampliar a 45 días más en casos especiales.

${ }^{11}$ Como ejemplo de esto, este caso es parte de la línea interpretación judicial del Fallo "SOLANO, MÓNICA INES C/ LIDERAR COMPAÑÍA GENERAL DE SEGUROS S.A. y otros S/ DESPIDO”, de septiembre de 2011. ${ }^{12}$ Algunas feministas liberales defensoras de una visión universal afirmaban que las licencias por maternidad debían regularse en el marco de las reglas generales de licencias por cuestiones médicas o de salud. En cambio, las feministas que proponían un trato especial afirmaban que tratar el embarazo o el parto como problemas médicos que justificarían una licencia estigmatizaría a las mujeres (Bergallo y Gherardi 2008).

${ }^{13}$ No obstante, los proyectos rara vez logran ser tratados en ambas Cámaras, perdiendo estado parlamentario tras dos años (Rodríguez Enríquez y Pautassi 2014).

${ }^{14}$ Para un análisis del sistema de asignaciones familiares ver: Bertranou y Bonari 2005; Hintze y Costa 2011; Arcidiácono et al. 2013, Pautassi et. Al. 2013b; entre otros.

${ }^{15}$ Un punto relevante en el marco de la discusión sobre las prestaciones y el principio de igualdad y no discriminación, es que desde 2009 se incorporó dentro del sistema de la seguridad social, la "Asignación Universal por hijo para la Protección Social" a los hijos de los trabajadores del sector informal y de las trabajadoras domésticas (Art. 4 , Resolución ANSES 393/09) que ganaran un sueldo inferior al Mínimo Vital y Móvil, y a los hijos de los desocupados y de los trabajadores temporarios registrados del sector agropecuario. Si bien se fueron creando nuevas asignaciones como la Asignación Universal por Embarazo que data de 2011 y es equiparable a la Asignación Prenatal, el resto de las asignaciones no tienen sus "espejos" en el sistema no contributivo. Uno de estos casos es justamente la Asignación por adopción, lo mismo sucede con la Asignación por nacimiento, por matrimonio, por escolaridad. 
Pilar Arcidiácono, Ana María Bestard, Rocío Riesco y Carla Zibecchi

${ }^{16}$ Art. 116 inc. d) ap. $7^{\circ}$ : "reintegro por guardería: el personal femenino tendrá derecho a esta asignación por cada hijo menor de cuatro años de edad que, por falta de cupo o inexistencia no pueda concurrir a guardería oficial gratuita....” En la misma ley se establece que la reglamentación determinará las condiciones exigidas para su otorgamiento. Ley Orgánica del Poder Policial No 11.501, sancionada en 1993.

${ }^{17}$ Condenas conminatorias de carácter pecuniario.

${ }^{18}$ Causas B 64.474 "Colegio de Abogados de la Provincia de Buenos Aires c/Provincia de Buenos Aires s/ amparo", sentencia del 19 de marzo de 2003 y "Sociedad de Fomento Cariló c/Municipalidad de Pinamar" sentencia del 29 de mayo de 2002. Asimismo este instrumento fue receptado en constituciones extranjeras y, dentro de la República Argentina, por la Constitución de la Provincia de Rio Negro.

${ }^{19}$ Ley de participación femenina en las unidades de negociación colectiva de las condiciones laborales (Cupo Sindical Femenino) No 25.674, sancionada en 2002.

${ }^{20}$ Investigaciones sobre el tema, han destacado los beneficios que pueden tener las políticas de conciliación familia y trabajo para sus empleados, empleadas y sus familias. (Rodríguez Enríquez 2010).

${ }^{21}$ Para un desarrollo de este tema ver en Rodríguez Enríquez y Pautassi (2014).

${ }^{22}$ Ley de Trabajo de las Mujeres y los Niños $N^{\circ} 11.317$, sancionada en 1924.

${ }^{23}$ Ley de Creación del Instituto Nacional de Jardines Maternales Zonales No 20.582, sancionada en 1973.

${ }^{24}$ En este contexto se enmarca, a modo de ejemplo, la nueva iniciativa gubernamental de la obligatoriedad de la sala de 4 años en el nivel inicial. 


\section{Referencias bibliograficas}

Albanese, S., coord. (2008) El control de convencionalidad. Buenos Aires: Ediar.

Arcidiácono, P. y Zibecchi, C. (2013) “¿Nuevas miradas a viejos problemas? El protagonismo del enfoque de derechos y el enfoque de género en las discusiones sobre pobreza y los programas sociales". Voces en el Fénix, 23:120-125.

Arcidiacono, P. y Gamallo, G. (2014) "Entre la confrontación y la funcionalidad. Poder Ejecutivo y Poder Judicial en torno a la política habitacional de la Ciudad de Buenos Aires". Revista Postdata. Revista de Reflexión y Análisis Político, 19:193-225.

Arcidiacono, P., Gamallo, G. y Straschnoy, M. (2013) “¿Consolidación de un sistema de protección social no contributivo en Argentina? El Plan Nacer, la Asignación Universal por Hijo y la Asignación por Embarazo". En Clérico, L., Ronconi, L. y Aldao, M. (Coords.), Tratado de Derecho a la Salud. Buenos Aires: AbeledoPerrot, 1387-1414.

Aulicino, C., Cano, E., DiazLangou, G. y Tedescchi, V. (2013) Licencias: Protección social y mercado laboral. Equidad en el Cuidado. Buenos Aires. Documento de trabajo del Área Protección Social de CIPPEC.

Bertranou, F. y Bonari, D., coords. (2005) Protección social en Argentina. Financiamiento, cobertura y desempeño, 1990-2003. Santiago de Chile: CEPAL.

Bidart Campos, G. (2004) El derecho de la Constitución y su fuerza normativa. Buenos Aires: Ediar.

CEPAL (2009). "Género, trabajo remunerado y no remunerado: eslabones en la discriminación y la desigualdad y Políticas Públicas y crisis de cuidado en América Latina: alternativas e iniciativas”. En CEPAL, Panorama Social en América Latina 2009. Santiago de Chile: CEPAL, 135-163.

CELS (2007) Derechos Humanos en Argentina. Informe 2007.Buenos Aires. Siglo XXI.

Courtis, C. y Bovino, A. (2001) “Por una dogmática conscientemente política”. En Christian Courtis (comp.), Desde otra mirada. Textos de teoría crítica del derecho. Buenos Aires: EUDEBA, 303-343.

Fairstein, C., Kletzel, G. y García Rey, P. (2010) "En busca de un remedio judicial efectivo: nuevos desafios para la justiciabilidad de los derechos sociales". En Arcidiáco- 
no, P., Espejo, N. y Rodríguez Garavito, C. (coords.) Derechos Sociales: justicia, política y economía en América Latina. Bogotá: Siglo del Hombre Editores,27-82.

Hintze, S. y Costa, M. I. (2011) “La reforma de las asignaciones familiares 2009: aproximación al proceso político de la transformación de la protección”. En Danani, Claudia y Hintze, Susana (coords.), Protecciones y desprotecciones: la seguridad social en la Argentina 1990-2010. Buenos Aires: Universidad Nacional de General Sarmiento,153-187.

Motta, C. y Sáez, M. (comps.) (2008) La mirada de los jueces. Género en la jurisprudencia latinoamericana. Tomo I. Bogotá: Siglo del Hombre Editores.

Pautassi, L. y Rico, N. (2011) "Licencias para el cuidado infantil. Derecho de hijos, padres y madres". Desafíos, Boletín de la infancia y adolescencia sobre el avance de Objetivos de desarrollo del MilenioSantiago de Chile: CEPAL-UNICEF. N 12:3-9.

Pautassi, L., Gherardi, N. y Zibecchi C. (2013a) "Presentación: El cuidado en la agenda pública”. En Pautassi, L. y Zibecchi, C. (Comps.) Las fronteras del cuidado. Agenda, derechos e infraestructura. Buenos Aires: Biblos, 9-26.

Pautassi, L., Arcidiácono, P. y Straschnoy, M. (2013b) Asignación Universal por Hijo para la Protección Social de la Argentina: entre la satisfacción de necesidades y el reconocimiento de derechos. Santiago de Chile: CEPAL, Serie Políticas Sociales, № 184.

Pautassi, L., Faur, E. y Gherardi, N. (2004) "Legislación laboral en seis países latinoamericanos. Avances y omisiones para una mayor equidad”, en: Serie Mujer y Desarrollo $N^{\circ}$ 56. Santiago de Chile: CEPAL.

Rodríguez Enríquez, C. y Pautassi, L. (coords.) (2014) La organización social del cuidado de niños y niñas. Elementos para la construcción de una agenda de cuidados en Argentina. Buenos Aires:ADC-CIEP- ELA (disponible en: http://elcuidadoenagenda.org.ar).

Rodríguez Enríquez, Corina (2010) “La organización del cuidado de niños y niñas en la Argentina y el Uruguay.” En S. Montaño Virreira y C. Calderón Magaña (coords.) El cuidado en acción: entre el derecho y el trabajo. Santiago de Chile: Cuadernos de la Cepal 94:15-140.

Rittich, Kerry (2012) "Feminización y contingencia: la regulación de las oportunidades laborales de las mujeres" en Natalia Gherardi (comp.) Justicia, Género y Trabajo. Buenos Aires: Red ALAS- Libraria:25-44. 
Staab Silke (2013) "Nuevas políticas de cuidado en Chile: expansión del cuidado infantil (2006-2010) y reforma de la licencia por maternidad (2011)", en Pautassi, Laura y Carla Zibecchi (comps.) (2013): Las fronteras del cuidado. Agenda, derechos e infraestructura. Buenos Aires: Biblos- ELA, 409-446.

Zelizer, V. (2009) “La intimidad en el Derecho", en La negociación de la intimidad. Buenos Aires: Fondo de Cultura Económica, 71-117.

Zibecchi, C. (2014) "¿Cómo se cuida en Argentina? Definiciones y experiencias sobre el cuidado de niños y niñas". Buenos Aires: ELA-ADC-CIEP (disponible en http://elcuidadoenagenda.org.ar). 